\title{
Lack of c-erbB-2 oncoprotein expression in male breast carcinoma
}

\author{
SB Fox, CA Day, S Rogers
}

\begin{abstract}
A series of 21 male breast carcinomas were immunostained using NCL-CB11, an antibody directed against the internal domain of the c-erbB-2 transmembrane oncoprotein. In contrast to female breast cancer, where up to $35 \%$ of cases show positivity, all of these cases were negative. This suggests that no prognostic information regarding patient survival can be made in these patients and that male breast carcinomas may be under different growth control mechanisms from female breast carcinomas.
\end{abstract}

The c-erbB-2 proto-oncogene is located on chromosome 17q21 and encodes an 18.5 kilodalton transmembrane protein of the tyrosine kinase family. ${ }^{1}$ C-erbB-2 shares extensive homology with the epidermal growth factor receptor ${ }^{1}$ and there is various evidence to suggest it too may also behave as a growth factor receptor. ${ }^{23}$ It is expressed in several types of human tumours including female breast carcinoma ${ }^{1}$ where amplification and c-erbB-2 oncoprotein expression are related to a poor prognosis. ${ }^{4}$

Male breast carcinomas, like their female counterparts, are thought to have hormonal influences in their aetiology. Associations with endocrine disease, hormone treatment, and altered oestrogen metabolism have been described. $^{56}$ These similarities between breast carcinomas of men and women warrant comparative investigations, not only to improve our understanding of this disease in men, but also to pinpoint distinctive features of female breast carcinoma. Studies in male breast carcinoma are few, however, due to the rarity of the disease. Indeed, we have been unable to identify any published data on oncoproteins in male breast carcinoma that are now known to be important in female breast carcinoma. Therefore, this study was undertaken to determine the frequency and distribution of the expression of one such oncoprotein, $\mathrm{c}-e r b \mathrm{~B}-2$, in male breast carcinomas and to evaluate any similarities with or differences from female breast cancers.

We examined a series of these lesions for c-erbB-2 expression using a mouse monoclonal antibody generated against the C-terminal portion of a synthetic peptide sequence of the internal domain (NCL-CB11; Novocastra Laboratories, Newcastle) on formalin fixed, paraffin wax embedded tissue. A previous report using this particular antibody on female breast carcinomas showed a high degree of correlation between c-erbB-2 amplification and c-erbB-2 oncoprotein expression. $^{7}$

Twenty one cases of male breast carcinoma were collected. One tumour was predominantly intraductal with a minor invasive component, the remainder being invasive ductal of no special type, although eight of these had a minor intraductal component. These were immunostained using the mouse monoclonal antibody NCL-CB11, using a peroxidase antiperoxidase technique. Endogenous peroxidase was blocked with $0.5 \%$ hydrogen peroxide in methanol before pre-treatment with rabbit serum at a 1 in 5 dilution for 15 minutes. The primary antibody was applied at a 1 in 40 dilution and incubated overnight at $4^{\circ} \mathrm{C}$ before application of rabbit anti-mouse immunoglobulin (Dako) at 1 in 100 for 30 minutes, followed by mouse peroxidase antiperoxidase (Dako) at 1 in 100 for 30 minutes. Peroxidase activity was visualised with diaminobenzidine solution. Omission of the primary antibody for each case was used as a negative control and a known c-erbB-2 positive Paget's disease of breast as a positive control. ${ }^{3}$ The positive control showed intense membrane staining of tumour cells in the nipple epithelium while no positive staining was identified in any of the sections of male breast carcinomas.

This study of a relatively large number of male breast carcinomas shows that, in contrast to female breast carcinomas which show up to $35 \%$ positivity, ${ }^{8}$ no significant c-erbB-2 expression is present and therefore amplification improbable. Thus unlike female breast carcinomas, no conclusion regarding its role as an independent negative predictor of prognosis can be made. As survival is little different between male and female breast carcinomas, ${ }^{6}$ similar expression of c-erbB-2 might have been expected, but these results suggest that other factors may be involved in the transformation and growth of male breast carcinomas.

We thank Dr PA Burton, Southmead Hospital, Bristol for supplying several cases.

1 McCann A, Dervan PA, Johnston PA, Gullick WJ, Carney DN. c-erbB-2 oncoprotein expression in primary human DN. c-erbB-2 oncoprotein expressi
tumours. Cancer 1990;65:88-92.

2 Heintz NH, Leslie KO, Rogers LA, Howard PL. Amplification of the c-erbB-2 oncogene and prognosis of breast tion of the C-erbB-2 oncogene and prognosis of breast

3 Kealing SI, Sinclair J, Wright C, et al. c-erbB-2 oncoprotein expression in mammary and extramammary Paget's disease: an immunohistological study. Histopathology 1990;17:243-7. 
4 Barnes DM. Breast cancer and a proto-oncogene. c-erbB-2 is a reliable prognostic marker. $\mathrm{Br}$ Med $\mathrm{J}$ 1989;299. 1061-2.

5 Rose DP. Endocrine epidemiology of male breast cancer (review). Anticancer Res 1988;8:845-50.

6 Korenman SG. The endocrinology of the abnormal male breast. Ann NY Acad Sci 1986;464:400-8.

7 Corbett IP, Henry JA, Angus B, et al. NCL-B11, a new monoclonal antibody recognising the internal domain of the c-erbB-2 oncogene protein effective for use on formalin-fixed paraffin-embedded tissue. J Pathol 1990;161:15-25.

8 Tsutsumi Y, Naber SP, DeLellis RA, et al. Neu oncogene protein and epidermal growth factor receptor are independently expressed in benign and malignant breast tissues. Hum Pathol 1990;21:750-8.

\title{
Tuberous sclerosis and parathyroid adenoma
}

\author{
L S Mortensen, J Rungby
}

\begin{abstract}
Very little is known about the pathogenesis of tuberous sclerosis. Over the past 10 years, however, increasing numbers of reports on adenomatous diseases in association with tuberous sclerosis have been published. A case of hypercalcaemia and parathyroid adenoma in association with tuberous sclerosis is presented, of which there has been one such report published previously.

This association might be another manifestation of this complex disease: it is therefore recommended that plasma calcium concentrations should be measured in such patients.
\end{abstract}

Tuberous sclerosis (also known as Bourneville's disease and epiloia) is a syndrome that is characterised by a triad of epilepsy, mental retardation, and cutaneous sebaceous adenomas. A variety of other clinical symptoms and subclinical abnormalities have also been described over the past decade. It is an autosomal dominant inheritance disease, not fully penetrant, with a high incidence of fresh mutations. ${ }^{1-5}$

In most populations the prevalence of tuberous sclerosis has been estimated to be about one in 100000 , but there are considerable regional differences (one in 20000 to one in $300000) .^{6-8}$ The basic metabolic mechanism underlying the disease remains unknown. Diagnosis can be made in utero.

Epilepsy, the most common clinical manifestation, is often present from early childhood, but not always. Mental retardation is found in about half the patients.

Sebaceous adenomas or cutaneous fibroangiomas are diagnostic and are found in up to $90 \%$ of the cases. The appearance varies and the most frequently affected areas are the nose, the chin, and the forehead. Hypopigmented areas are often a concomitant feature.

The central nervous system abnormalities in tuberous sclerosis are characterised by calcified subependymal and cortical nodules (tubers). Intracranial gliomas and retinal or optical phacomas are also often found. The skeleton is often affected. Characteristically, sclerotic areas in the calvarium, pelvic bone, and columna are present. The metacarpals and metatarsals display periostal thickening and cysts. Other organs have been reported as being affected: renal angiomyolipomas or cysts are not uncommon, as are cardiac rabdomyomas. Pulmonary cysts may also be evident. ${ }^{1-10}$

Although apparently rare, glands have also been affected in some cases: hepatic angiomyolipomas, islet-cell adenomas of the pancreas, adrenal adenomas, pituitary adenomas, a few reports of precocious puberty, and disease of the testes, ovaries, and thyroid gland. ${ }^{1389}$

We are aware of only one previous description of parathyroid disease in a patient with tuberous sclerosis. ${ }^{10}$ We report the second published case of primary hyperparathyroidism associated with tuberous sclerosis.

\section{Case report}

A 15 year old boy was admitted to hospital with abdominal pains. He had no history of endocrine disease. One of his sisters had confirmed tuberous sclerosis comprising epilepsy, mental retardation, and cutaneous adenomas. Our patient also had cutaneous sebaceous adenomas, and tuberous sclerosis was suspected; the family history was then obtained. The mother was subsequently found to have tuberous sclerosis; she had typical cutaneous signs but no other symptoms, and she was normocalcaemic.

Acute pancreatitis and hypercalcaemia was diagnosed in our patient. Further examination showed biochemical hyperparathyroidism, with an eight-fold increase in parathyroid hormone activity (intact PTH) (using an immunoreactive $\mathrm{PTH}$ assay measuring the intact PTH molecule, normal range 10-65 ng/1.) Combined thallium-technetium scintigrams and subsequent ultrasonography indicated a parathyroid adenoma located on the right side of the neck, just above the clavicle. A parathyroid adenoma was removed and the diagnosis was verified microscopically. After surgery calcium metabolism returned to normal along with intact plasma PTH. Tuberous sclerosis was diagnosed because of dermatological changes, sebaceous adenomas of the face, and hypopigmented areas on both 